\title{
THE COVERING HOMOTOPY THEOREM
}

M. L. CURTIS

William Huebsch [1] has proved that the covering homotopy theorem (see Steenrod [2, p. 54]) for locally trivial fiber spaces holds for the class of paracompact spaces. In this note we show that in quite general circumstances (base space and total space metric) the covering homotopy theorem holds for the class of all topological spaces. One cannot use the usual type of construction because, as Huebsch has shown, that construction characterizes paracompact spaces.

Given $p: E \rightarrow B$ we construct a test space as follows. Let $\tilde{E}=\{f: I \rightarrow B\}$ topologized with the $c-0$ topology ( $I$ is the unit interval). Let $C$ be the subset of $\tilde{E} \times E$ defined by

$$
C=\{(f, e) \mid f(0)=p(e)\} .
$$

Next we define maps

$$
\begin{aligned}
\beta: C & \rightarrow E \text { by } \\
\beta(f, e) & =e
\end{aligned}
$$

and

$$
\begin{aligned}
& \Delta: C \times I \rightarrow B \text { by } \\
& \Delta(f, e, t)=f(t) .
\end{aligned}
$$

Now $C$ will be a test space in the following sense.

Proposition. If $(E, B, p)$ has the covering homotopy property for $C$, then it has the covering homotopy property for any topological space.

PROOF.

$$
\begin{aligned}
\beta: C & \rightarrow E, \\
\Delta: C \times I & \rightarrow B
\end{aligned}
$$

is a covering homotopy situation since

$$
\begin{aligned}
p \beta(f, e) & =p(e), \\
\Delta(f, e, 0) & =f(0),
\end{aligned}
$$

and

$$
p(e)=f(0) \text { since }(f, e) \in C .
$$

Presented to the Society, September 1, 1955; received by the editors July 6, 1955. 
Hence by assumption there exists an extension

$$
\Gamma: C \times I \rightarrow E
$$

of $\beta$ such that

$$
p \Gamma=\Delta .
$$

Now let $X$ be a topological space and suppose we have a covering homotopy situation

$$
\begin{aligned}
\phi: X \rightarrow E, & \\
\psi: X \times I \rightarrow B, \quad p \phi & =\psi_{0} .
\end{aligned}
$$

Let $f_{x}$ denote the path $\psi \mid x \times I$ and define

$$
\begin{gathered}
\alpha: X \rightarrow C \text { by } \\
\alpha(x)=\left(f_{x}, \phi(x)\right) . \\
\left(f_{x}, \phi(x)\right) \in C \text { since } p \phi(x)=\psi_{0}(x)=f_{x}(0) .
\end{gathered}
$$

Define

$$
A: X \times I \rightarrow C \times I
$$

by

$$
A(x, t)=\left(f_{x}, \phi(x), t\right)
$$

Now

$$
\beta \alpha=\phi
$$

since

$$
\beta \alpha(x)=\beta\left(f_{x}, \phi(x)\right)=\phi(x)
$$

Also

$$
\Delta A=\psi
$$

since

$$
\Delta A(x, t)=\Delta\left(f_{x}, \phi(x), t\right)=f_{x}(t)=\psi(x, t) .
$$

We must simply check that $\Gamma A: X \times I \rightarrow E$ covers $\psi$; i.e. that $p \Gamma A=\psi$.

$$
p \Gamma A(x, t)=p \Gamma\left(f_{x}, \phi(x), t\right)=\Delta\left(f_{x}, \phi(x), t\right)=f_{x}(t)=\psi(x, t) .
$$

This proves the proposition.

Corollary. Suppose $(E, B, p)$ has the covering homotopy property with respect to $C$. Once $\Gamma$ is chosen there is a unique covering homotopy for every covering homotopy situation involving $(E, B, p)$. 
ThEOREM. Let $(E, B, p)$ be a locally trivial fiber space and suppose $C$ is paracompact. Then $(E, B, p)$ has the covering homotopy property with respect to all topological spaces.

Proof. This follows immediately from the result of Huebsch and the proposition given above.

CoRollary. Let $(E, B, p)$ be a locally trivial fiber space and suppose $E$ and $B$ are metric spaces. Then $(E, B, p)$ has the covering homotopy property with respect to all topological spaces.

Proof. Since $B$ is metric, so is $\tilde{E}$ and hence so is $\tilde{E} \times E$ and so is $C$. A. H. Stone [3] proved that a metric space is paracompact, so we can apply the theorem above.

\section{BIBLIOGRAPHY}

1. William Huebsch, On the covering homotopy theorem, Ann. of Math. vol. 61 (1955) pp. 555-563.

2. N. E. Steenrod, The topology of fiber bundles, Princeton University Press, 1951.

3. A. H. Stone, Paracompactness and product spaces, Bull. Amer. Math. Soc. vol. 54 (1948) pp. 977-982.

NORTHWESTERN UNIVERSITY 